\title{
Effects of Domestic Violence on Children's Education: The Case Study of Mpemba, in Blantyre District [Malawi]
}

Phillip G Kanchiputu* and Marisen Mwale

Department of Psychology, Mzuzu University, Malawi

\begin{abstract}
The main objective of the study was to examine the effects of domestic violence on children's education. The study attempted to expose causes, kinds and perpetrators of domestic violence against children, and how the violence negatively affects schooling victims. The study used both qualitative and quantitative paradigms in which data was collected through questionnaires, oral interviews and focus group discussion. The study sampled adolescent students and adults in selected schools and villages around Mpemba area, in Blantyre, a district in southern Malawi. The research depicted negative impacts of domestic violence against children on the victim's education. It unveiled that domestic violence against schooling children harms them emotionally, psychologically and physically. In the long run, this harm hinders the victims' learning process in many ways such as inflicting the victim to lose interest in education. The research adds knowledge to the awareness of the relationship between domestic violence against children and victims' education. This project will contribute to future research on similar topics.
\end{abstract}

Keywords: Domestic violence; Domestic violence against children; Exposure to domestic violence; Victim

\section{Introduction}

Most contemporary scholars agree that domestic violence is one of the negative practices that mostly happen in households. Gordon [1] described the term domestic violence as generally understood to apply to any assault committed by a person who either shares a living arrangement with or is involved in an intimate relationship with the victim of the assault. Domestic violence is a bad practice which can affect children's education in one way or the other. Anyone can be a victim. Domestic violence can impact anyone regardless of their gender, race, age, culture or religion. However, most often women and children are the victims of domestic violence. Most studies focused on women as victims of domestic violence but this study dwelled on children as victims of domestic violence. It can occur in many forms including emotional abuse, psychological violence, neglect, physical assault, witnessing (exposure to) physical violence and sexual assault. Children exposure to this form of violence has considerable potential to be perceived as life threatening by those victimized and leave them with a sense of vulnerability, hopelessness, psychological disorders and emotional threats. Which end up affecting their academic lives [2,3].

Domestic violence is common in male dominated cultures such as patrilineal and patriarchy cultures, as it is justified in customs and traditions, and condoned by law. Some of the domestic violence cases go unreported, since children may suffer in silence [4].There are various reasons for lack of reporting, for example, young children lack capacity to report, many children are afraid of reprisals by perpetrators or interventions by the authorities that worsen their overall situation and parents, the primary protectors of children, are in many cases also the perpetrators of violence and may remain silent if the violence is perpetrated by other family members or powerful members of the community or society.

Violence against children in the home and family has been condemned by many organizations. For example, the United Nations signed a charter aimed at protecting children's rights in 1989 and it is called the United Nations Convention on the Rights of the Child (UNCRC) (www.unicef.org/crc/). And African countries also took an initiative in protecting children rights, with the African Charter on the Rights and Welfare of the Child (ACRWC), it is also called the Children's Charter, adopted by the Organization of African Unity (OAU) in 1990, (www.pulp.up.ac.za/../2009-11.pdf).
Fantuzzo and Mohr [2] conducted a study on the prevalence and effects of child exposure to domestic violence. The study was carried through telephone surveys and questionnaires. They categorized crimes that pertain to domestic violence as include: assault offenses (aggravated assault, simple assault and intimidation), forcible rape and non-forcible rape, disorderly conduct and family offenses. They also said that children exposed to domestic violence differ from nonviolent homes with respect to one or more aspects of child functioning, including: externalizing behaviors (such as aggressive behavior and conduct problems); internalizing behaviors (such as depression, anxiety and low self-esteem); intellectual and academic functioning; social development, and physical health and development, (www. princeton.edu/futureofchildren/publications/../09-1.pdf). The study mentioned one of the impacts of domestic violence against schooling children as intellectual and academic functioning but it did not explain how domestic violence affects children in intellectual and academic functioning.

Another study conducted by African Child Policy Forum (ACPF) (2011) shows the prevalent of violence against children in different forms of physical, psychological and sexual violence and also the study found fathers or male relatives to be the most perpetrators of domestic violence (www.africanchildforum.org/site/.../ACPF-violence-againstchildren.pdf). But the study did not tackle the effects of the violence on the academic life of the victims (children). Another study by Edleson [3] found that children's problems associated with witnessing violence can be grouped into behavioral and emotional problems, and cognitive functioning and attitude problems. On behavioral and emotional problems; the study found that children exposed to domestic violence exhibits more aggressive and antisocial (externalized behaviors) as well as fearful and inhibited behaviors (internalized behaviors) and show lower social competence than other children. On cognitive functioning

*Corresponding author: Phillip G Kanchiputu, Department of Psychology, Mzuzu University, Malawi, Tel: +265 1320 722; E-mail: marisen.mwale@yahoo.co.uk

Received July 22, 2016; Accepted August 17, 2016; Published August 22, 2016

Citation: Kanchiputu PG, Mwale M (2016) Effects of Domestic Violence on Children's Education: The Case Study of Mpemba, in Blantyre District [Malawi]. J Psychol Abnorm 5: 152. doi: 10.4172/2471-9900.1000152

Copyright: ( 2016 Kanchiputu PG, et al. This is an open-access article distributed under the terms of the Creative Commons Attribution License, which permits unrestricted use, distribution, and reproduction in any medium, provided the original author and source are credited. 
and attitude problems; the study found that increased violent exposure was associated with lower cognitive functioning. And also children's (especially adolescent boys) exposed to adults' domestic violence may generate attitudes justifying their own use of violence (that acting aggressively enhances one's reputation or self-image), (www.vawnet. org). However the study did not reach to the point of describing impacts of behavioral and emotional problems, and cognitive functioning and attitude problems in relation to children's education. Moreover, Worrall et al. [5] argued that the social and educational development of some children may be impaired by the support they give to the abused parent and girls, in particular, may be anxious to protect their mother and younger siblings, even where this involves placing themselves at physical risk (www.scie.org/.../briefing25.pdf). McKinney Vento Law [6] states that children and youth who flee violent homes with a parent survivor face many heightened risks for emotional and behavioral problems. They may be more likely than their peers to experience or to participate in emotional or physical abuse themselves. These effects can have a pronounced impact on children's adjustment in school, including their ability to learn and their concentration levels (www.center.serve. org/nche/downloads/briefs/domestic.pdf).

From the studies noted above, some studies focus on the forms of domestic violence and its effects on children's wellbeing, thereby creating a gap which has been fulfilled by this study through studying effects of domestic violence on children's education. On the other hand, other studies noted above partially touched the impacts of domestic violence on children's academic lives. So, this study has expanded fully their points in reference to the current state of affairs on the ground by examining the effects of domestic violence on children's education.

\section{The Research Setting}

The research was carried out around Mpemba area, Traditional Authority Somba, in Blantyre district which is located in southern Malawi. Mpemba is a rural area where the majority of the population is small scale farmers. The community is patriarchal in culture and the area registers high rates of domestic violence statistically on a national scale.

\section{Materials and Methods}

The study was a case study. It triangulated quantitative and qualitative paradigms in designing, sampling, data collection and analysis. By combining these research paradigms the purpose was to overcome the weaknesses and problems that come from using a single paradigm. Thus, triangulation increased the credibility and validity of the results.

The respondents who participated in the study were parents from Somba village; officers from Mpemba Police Unity; primary school adolescents from Mpemba Reformatory Centre; and then secondary school adolescents and teachers from three schools namely: Mpemba Community Day Secondary School, Mpemba Private Secondary School and Nankumba Community Day Secondary School. The researcher targeted Mpemba police officers to find out whether domestic violence cases have been reported to them and who was reporting. And Mpemba Reformatory Centre was chosen to find out if some of the children being kept there were victims of domestic violence before. These respondents were selected for convenience purposes since it was deemed easier for the researcher to walk or cycle from home to the sites.

The sample for this research was 60 . These involved 15 teachers (5 from each school) and 15 students, 5 police officers from Mpemba Police Unity, 10 parents and 15 children from Mpemba Reformatory
Centre. This sample was chosen simply because it was the number that the researcher would manage in terms of resources. The sample was not necessarily meant to be representative of the entire population.

The respondents in the study were chosen using both systematic and convenience sampling techniques based on the availability of the respondents during the data collection period. Systematic sampling was used to collect data from the students in which sample members from a class were selected according to a random starting point and a fixed, periodic interval. And also convenience sampling was used to collect data from teachers, police officers and parents, since they were chosen based on availability, time, location or ease of access.

Questionnaires, oral interviews and focus group discussion were used as instruments for data collection. The questionnaire was preferred for its suitability to this study. It was suitable as a method of data collection because it allows the researcher to reach a larger sample within limited time. Interviews were preferred because they help to enlist the cooperation of respondents and establish a rapport with them. Face to face interviews allow the interviewer to deeply explore the respondents' feelings and perspectives on a subject under study without the influence of peers. And finally, focus group discussions were chosen to allow for a more extensive exploration of the topic under study. Focus group discussions complemented the questionnaire and allowed the researcher to probe more into the issue at hand from the primary respondents. The three instruments, that is, questionnaire, interviews and focus group discussions were used in the study for the purpose of cross-triangulation and confirming information collected from various respondents.

\section{Results and Discussion}

\section{Kinds of domestic violence against children}

The study results show, $100 \%$ of students and $88.2 \%$ of adults pointed out that domestic violence exists in their community and they know it. It is pertinent to highlight that some cultures have higher rates of cases nationally than others with patriarchal cultures having significantly higher rates statistically than matrilineal cultures. A majority of communities [more than 90\%] around the research site are patriarchal and therefore it is not surprising that they were more likely to register higher cases of domestic violence. Results collected through questionnaires, interviews and focus group discussions show that students and adults have different but similar ideas on how they describe domestic violence. When the concept of domestic violence was explored from both students and adults, some of the ideas that emerged include: "Any incident of threatening behavior or abuse between people who are family members," a 16 years old girl. The respondents' description of domestic violence against children embodies some critical ideas such as abuse, aggressive behavior, violation of human rights, rough treatment, confrontation and misunderstanding in the households and families. The respondents' description of domestic violence is in line with Gordon [1] who described domestic violence as any assault committed by a person who either shares a living arrangement with or is involved in an intimate relationship with the victim of the assault.

Findings show that $90 \%$ of learners have experienced domestic violence at a certain point in their lives. And $88.2 \%$ of adults pointed out that sometimes children are exposed to domestic violence in the households. Since the majority of students reported to be victims of domestic violence and at the same time the majority of adults reported that children are sometimes mistreated in the homes, in this regard it can be noted that domestic violence is happening on the ground. 
Moreover, students who have experienced domestic violence and adults who knew domestic violence indicated the forms of violence experienced by children. The kinds of domestic violence against children reported include; firstly, emotional violence which was a most common reported form of violence against children. The respondents gave examples of emotional violence such as insults, shouts, repeated criticism, frequent blames, threatening, denying of love and affection, and ignoring. This simply highlights that in homes children are exposed to words and actions that make them feel useless or worthless. Adults use abusive language in order to make children obey their commands. This violence harms children's feelings and thinking. Secondly, physical violence is another form of domestic violence cited by participants. Students and adults reported that children are sometimes exposed to physical violence in the homes. Respondents were able to explain this violence through examples. The examples given are: beating (slapping or kicking), pushing and assault with an object or weapon. This violence harms or pains children physically and emotionally at same time. Thirdly, other respondents reported experiencing economic or material abuse. Some students pointed out that they do experience economic violence in their homes and also adults mentioned that children are exposed to economic violence. The participants gave examples of economic violence such as destruction of a child's personal property, withholding of financial support like not providing school fees or uniform, giving a child excessive labour and denying food to a child. Respondents said that this violence may happen as form of punishment or it may be due to other unknown reasons in the perpetrator's mind while other students reported being given excessive work in order to be given food or to be recognized as a family member. In addition, some students reported their school text or exercise books being burnt as a form of punishment to them. The examples given simply show that children are facing economic violence in the households and it negatively affects their wellbeing. Fourthly, sexual violence is another reported form of domestic violence against children. Respondents expressed that some children are experiencing sexual violence in the households. These participants said that children are exposed to sexual violence such as touching of body sexual organs and kissing on the mouth, against their will. A 15 years old girl during the interview reported to be a victim of sexual abuse. She said "My uncle always forcefully touches my breasts, my buttocks and kissed me on the mouth. And I am disappointed" This testimony simply shows that children are victims of sexual violence in the homes and it negatively affects their feelings and thinking. Fifthly, respondents reported another form of violence which is witnessing physical violence. Participants said that sometimes children observe and hear their parents or guardians fighting or insulting at each other. During the focus group discussion a 14 years old boy said "It hurts me because my father frequently beats and insults my mom." This simply indicates that observing parental physical and verbal violence can negatively affects the child. Of all these forms of violence the highest reported is emotional violence and the least reported is witnessing physical violence, representing $40 \%$ and $3.3 \%$ respectively. These findings concur with findings documented by research $[3,4,7]$ which found that children are exposed to domestic violence such as emotional, physical, sexual and economic violence. In the respect of the study results it can be pointed out that children are experiencing different forms of domestic violence in their homes.

The study results also show that the perpetrators of violence vary according to whom one is staying with at that moment. More students reported experiencing violence perpetrated by their mothers. This is so maybe because children spent more time with their mothers at home. Other perpetrators reported by students include; stepmothers, fathers, stepfathers, uncles, aunt and finally, sister in law (brother's wife). In this regard the highest perpetrator is mother and the least perpetrator is sister in law, representing 30\% and 3.3\%, respectively. However, by going through a specific gender, it shows that the gender of the perpetrator varies according to the gender of the victim. Highest number of girls reported experiencing violence perpetrated by mothers while more boys reported experiencing violence perpetrated by their fathers. This is line with Lindon [8] who found that mothers are the most perpetrators of violence against girls while fathers are the most perpetrators of violence against boys. Therefore, the most perpetrator of violence against a girl child is the mother and for a boy child is the father.

Findings show that $80 \%$ of students who experienced violence did not report the cases of domestic violence against them. During the focus group discussion and interviews the students said that they do not report cases of domestic violence because they fear the potential consequences, for instance, they fear that the violence may worsen, others fear to be abandoned or rejected as a family member by their parents or guardians. While others said they never thought of reporting cases of domestic violence against them. Respondents said those who report in most cases do so after being directed or advised by an adult person. This concurs with Newell [4] who pointed out that only a small proportion of all acts of violence against a child are reported and investigated. He said that the reasons for lack of reporting are that children lack capacity to report and also children are afraid of reprisals by perpetrators. In this case, therefore, it can be deduced that children do not report domestic violence because they fear the potential consequences and others lack capacity to report.

\section{Causes of domestic violence against children}

Both groups of students and adults gave various suggestions on the causes of domestic violence against children in the households. More respondents (40\%) reported powerless status of children as the main cause of domestic violence against them in the households. Their low social status in the hierarchy of power in the society left children prone to domestic violence. Thus, power based on parent-child relationship. Children are being violated just because they are children. Secondly, respondents reported poverty as a cause of domestic violence against children. They said that poor living conditions create pressure on the family and stress on the parenting of that family; this may lead to domestic violence. Thirdly, respondents reported drug and alcohol abuse as a cause of violence. They said drug and alcohol abuse may drive the drunkard on the creation of poor relationship between parents (or guardians) and children, in the long run, this may lead to domestic violence against children. Fourthly, respondents mentioned child's misbehavior as a cause of domestic violence since adults may apply excessive punishment or rough treatment in order to put the bad behavior under control. Apart from the above points, respondents also mentioned other causes of domestic violence like jealousy and parent's ignorance. Of these points, the highest reported cause was powerless status of children and least reported was child's misbehavior, representing $40 \%$ and $5 \%$ respectively. These points concur with Gordon [1] who identified drug abuse and alcoholism, poverty, and age and gender as causes of domestic violence.

\section{Effects of domestic violence on schooling children}

All respondents, thus $100 \%$ of students and adults conceded that domestic violence against children negatively affects education of the victim (child) in one way or the other. The respondents went further by stating various ways in which domestic violence against children affects the victims' education. Domestic violence such as beats, insults, shouts, ignoring, denying food, sexual harassment, destruction of child 
personal property, threats and humiliation, among others may result in psychological harm, emotional harm, physical pain or injury, lack of self-confidence and lack of self-esteem on the victim (a child). These states of affairs have great negative impact on the victim's learning process since it affects the well-being of the particular victimized child. In addition, more respondents (41.2\%) said that domestic violence against children affects the victim's education in the sense that it influences the abused child to lose interest in education. Participants added by saying that the pain or harm inflicted on children by violence may affect their emotional, psychological and physical well-being and these conditions will as a result shift child's focus on education to a worrisome state over one self's life. The second reported effect of domestic violence on schooling children is that it sometimes causes the victimized child to arrive late at school. On this note, some students said that they had to do a certain work before going to school in the morning and these pieces of work often times made them to arrive late at school. The reported pieces of work range from house chores (such as fetching water, cooking or sweeping) to field work. Thirdly, students' absenteeism from school is another reported effect of domestic violence on schooling children. Respondents said that child abuse such as excessive labour, verbal aggression, food denial and physical harm can result in influencing a victimized child to be absent from school. More importantly, some parents took their children with them to piece works instead of letting them go to school. Furthermore, dropping out of school is one of the reported effects of domestic violence on schooling children. Respondents reported that violence like withholding financial support such as not providing school fees, uniforms and other essential things may force the victimized child to drop out of school. Another reported effect is changing of schools. The participants mentioned that when parents separate due to some misunderstanding in the households some children move with the other parent to another place, this results in forcing concerned student to transfer to a school located in the new area of residence. Moreover, respondents mentioned early marriage as an effect of domestic violence on schooling children. They said that some parents force their children to marry while young on the expense of going to school. Not only that but also emotional harm, psychological harm and physical pain inflicted to them by domestic violence, may influence a girl child in dilemma to opt for marriage as relief from a violent home. These instances will automatically force the victim to drop out of school for marriage. During the interview, a 34 years old woman gave testimony by saying that "I opted for marriage as relief from my stepmother's evil hand though I was young by then." The highest reported effect of domestic violence on schooling children is loss of interest in education and the least reported effect is early marriage, representing $41.2 \%$ and $5.9 \%$. This is line with findings documented by research $[2,5,6]$, which found that domestic violence has a pronounced negative impact on children's adjustment in school, including their ability to learn and their academic concentration levels thereby impairing social and educational development of victimized children which may result in unsatisfactory intellectual and academic functioning. With regard to the views expressed above, it can be deduced that domestic violence affects education of the victimized schooling child.

\section{Suggested prevention measures of domestic violence}

Findings show that the respondents suggested various prevention measures for domestic violence against children. More students (40\%) said that parents should be educated on children rights in order to prevent domestic violence against children. The students expressed that civic educating or sensitizing parents on children's rights can make them aware that children also deserve dignity as adults. And also sensitization of parents may influence adults to change from their bad parental styles to safe parental styles. Secondly, some students suggested that the community should have a place responding to domestic violence against children were victimized children can go to seek help. Moreover, students suggested that local leaders should formulate bylaws aiming at preventing domestic violence against children. Another suggestion from the students was that the police should arrest the perpetrators of domestic violence against children. And lastly, the least students (10\%) said that they have no idea on what should be done to prevent domestic violence against children. On the other hand, more adults (45\%) suggested that parents or adults should be held accountable for their children but need not to be arrested. The second suggestion from adults was that parents need to be sensitized on children rights for them to know that a child is a person like them who deserves respect. Furthermore, the other suggestion from adults was that police suggestion boxes should be introduced in communities so that victimized children would throw in their concerns. And finally, other parents were of the view that nothing should be done to parents or guardians because parents know what is best for their children and also nothing should be done to adults because anyone can make mistakes. This is also consistent with findings by Naker [7] in studies conducted in Uganda where it was found that prevention measures of domestic child abuse include that parents should be held accountable over their children; police should arrest perpetrators of violence, and the community should have a place responding for the victimized children.

\section{Conclusion}

As reflected in the current study, 80 to $100 \%$ of respondents reported that domestic violence exists in the community; students reported that they do experience domestic violence in their homes; and adults reported that sometimes adults mistreat children in several ways. Respondents mentioned forms of domestic violence against them as physical violence, emotional violence, sexual violence and economic violence. The reported violence perpetrators include: mothers, stepmothers, fathers, stepfathers and uncles. Causes of domestic violence given are as follows; poverty, jealousy, drug and alcohol abuse, child's misbehavior and child's low social status in the hierarchy of power in the community. Findings shows that domestic violence inflicts harm to children such as emotional harm, psychological harm, physical pain and low self-esteem which impact the child learning systems thereby influencing a child to lose interest in education, arrive late at school, being absent from school, dropout of school and even early marriage. Since the purpose of the study was to examine the effects of domestic violence on children's education, the research study results, therefore, have revealed that domestic violence against children affects the education of schooling victim (child). More research however needs to be done on strategies that can be put into place to mitigate the underlying causal factors of this social pathology considering the implications it has not only on children but society at large.

\section{References}

1. Gordon LP (Ed.) (2002) Violence against women. New York : Novinka Books.

2. Fantuzzo JW, Mohr WK (1999) Prevalence and effects of child exposure to domestic violence.

3. Edleson JL (1997) Problems associated with children's witnessing of domestic violence.

4. Newell P (2008) Challenging violence against children: Handbook for NGOs working on the follow-up on the UN study. London: Save the Children Alliance.

5. Worrall A, Boylan J, Roberts D (2008) Children's and young people's experiences of domestic violence involving adults in a parenting role.

6. McKinney Vento Law. (2001) Domestic violence, homelessness and children's education. 
Citation: Kanchiputu PG, Mwale M (2016) Effects of Domestic Violence on Children's Education: The Case Study of Mpemba, in Blantyre District [Malawi]. J Psychol Abnorm 5: 152. doi: 10.4172/2471-9900.1000152

7. Naker D (2005) Violence against children: The voices of Ugandan children and adults.

8. Lindon J (2008) Safeguarding children and young people: Child protection 0-18 years. London: Holdder Education.

9. African Child Policy Forum (ACPF) (2011) Violence against children in Africa.

10. Education in Malawi, world camp and educational improvement.

11. Mantell A, Scragg T (2009) Safeguarding adults in social work. Glasgow: Learning Matters.
12. Munro E (2007) Child protection. London: Sage Publications.

13. Newman DM (2008) Sociology: Exploring the architecture of everyday life. Los Angles: Pine Forge Press.

14. UNICEF Malawi Child Protection Strategy 2012-2016.

15. Wolfe DA, Jaffe PG (1999) Emerging strategies in the prevention of domestic violence.

16. www.pulp.up.ac.za/../2009-11.pdf

17. www.unicef.org/crc/ 